\title{
Physiological System Identification with the Kalman Filter in Diffuse Optical Tomography
}

\author{
Solomon Gilbert Diamond ${ }^{1}$, Theodore J. Huppert ${ }^{1}$, Ville Kolehmainen ${ }^{3}$, \\ Maria Angela Franceschini ${ }^{1}$, Jari P. Kaipio ${ }^{3}$, \\ Simon R. Arridge ${ }^{2}$, and David A. Boas ${ }^{1}$ \\ 1 Massachusetts General Hospital, Martinos Center for Biomedical Imaging ${ }^{\star}$, \\ Charlestown MA 02129, USA \\ sdiamond@nmr.mgh.harvard.edu \\ http://www.nmr.mgh.harvard.edu/PMI \\ 2 Department of Computer Science, University College London, London, UK \\ 3 Department of Applied Physics, University of Kuopio, Kuopio, Finland
}

\begin{abstract}
Diffuse optical tomography (DOT) is a noninvasive imaging technology that is sensitive to local concentration changes in oxyand deoxyhemoglobin. When applied to functional neuroimaging, DOT measures hemodynamics in the scalp and brain that reflect competing metabolic demands and cardiovascular dynamics. Separating the effects of systemic cardiovascular regulation from the local dynamics is vitally important in DOT analysis. In this paper, we use auxiliary physiological measurements such as blood pressure and heart rate within a Kalman filter framework to model physiological components in DOT. We validate the method on data from a human subject with simulated local hemodynamic responses added to the baseline physiology. The proposed method significantly improved estimates of the local hemodynamics in this test case. Cardiovascular dynamics also affect the blood oxygen dependent (BOLD) signal in functional magnetic resonance imaging (fMRI). This Kalman filter framework for DOT may be adapted for BOLD fMRI analysis and multimodal studies.
\end{abstract}

\section{Introduction}

Diffuse optical tomography (DOT) is a noninvasive imaging technology that uses near infrared (IR) light to image biological tissue. The dominant chromophores in this spectrum are oxyhemoglobin (HbO), deoxyhemoglobin (HbR), lipids and water. The basis of DOT is in vivo near infrared spectroscopy of these dominant chromophores in the tissue. Tomographic images in DOT are constructed by simultaneously measuring from many local regions that cover a larger volume of tissue. The in-plane resolution limit of DOT increases rapidly with depth because biological tissue is a highly scattering medium for near infrared light. This diffuse property of the light also limits the penetration depth in adult human

\footnotetext{
* This research was funded by NIH T32-CA09502, P41-RR14075, R01-EB001954 and the MIND Institute.
} 
brain imaging to about $3 \mathrm{~cm}$, which is sufficient to study most of the cerebral cortex. See Gibson et al. for a complete description of DOT [1]. Clinical and research applications of DOT arise due to is its specificity to the physiologically relevant chromophores $\mathrm{HbO}$ and $\mathrm{HbR}$. Potential clinical and research applications for DOT abound in brain injury, degenerative neurovascular diseases and in cognitive neuroscience. Other research areas for DOT include fetal and neonatal monitoring and breast cancer detection. DOT is particularly suitable for in situ monitoring and multi-modal imaging [2]

The dynamics measured with DOT in the functional neuroimaging application are caused by local changes in blood volume and oxygenation in the scalp and in the brain. Due to the physical constraints of noninvasive imaging with DOT, the scalp and brain effects are combined in the measurements. The measured hemodynamics are caused by blood pressure regulation, cerebral blood flow autoregulation, local vasomotion and the vascular response to neuronal activity. Complexity arises because of interactions between these factors. The primary aim of DOT functional neuroimaging is to separate the stimulus related brain function signal from the background physiology related signal. The main problem is that the latter of these two is much stronger. A method to help resolve the physiological components in DOT is to include noninvasive auxiliary physiological measurements in the analysis. Many instruments can be used during DOT experiments. Examples are the blood pressure monitor, pulse oximeter, electrocardiogram (ECG), chest band respirometer, spirometer and capnograph. A further complexity of DOT analysis is that even when auxiliary physiology is included in the analysis, their effects do not appear to be stationary in time or space. We commonly observe that signal dynamics that are correlated with respiration, for example, will vary significantly in amplitude and relative phase angle at different measurement locations even when breathing rate and depth are held constant. The present objective is to separate the physiological components of DOT with a dynamical model.

State-space estimation has previously been applied to DOT without physiological regressors 3. Prince et al. 4 fit the amplitude and phase angle of three non-stationary sinusoids to DOT time-series data using the Kalman filter. While supporting the principle of using the Kalman filter in DOT analysis, the three-sinusoid model does not allow for the most commonly used event related experimental designs nor can it use readily available physiological measurements such as blood pressure as a regressor. Zhang et al. used principal component analysis (PCA) to reduce the background physiological variance in functional neuroimaging experiments [5]. Anecdotal evidence was presented that certain principal components correlate with blood pressure and respiratory dynamics. This observation of statistically uncorrelated blood pressure and respiratory dynamics contradicts known respiratory interactions in blood pressure regulation 6]. Due to physiological interactions, the orthogonal projections in PCA are more likely to be mixtures of physiological effects. Standard linear regression methods in fMRI analysis [7] accept multiple regressors that could easily include auxiliary physiological measurements but will not accommodate temporal non- 
tration changes in $\mathrm{HbO}$ and $\mathrm{HbR}$. In order to perform this convolution within a Kalman filter framework, it is convenient to define auxiliary states $\mathbf{z}$ that merely store a regression length $n_{r}$ of most recent inputs. The measurements $\mathbf{y}$ are the time-series of changes in optical density $(\Delta \mathrm{OD})$ for each source-detector pair and wavelength. This discrete-time process can be described as

$$
\begin{aligned}
\mathbf{x}_{k} & =\mathbf{A} \mathbf{x}_{k-1}+\mathbf{w}_{k-1} \\
\mathbf{z}_{k} & =\mathbf{B} \mathbf{z}_{k-1}+\mathbf{C} \mathbf{u}_{k} \\
\mathbf{y}_{k} & =\mathbf{D}\left(\mathbf{z}_{k}\right) \mathbf{x}_{k}+\mathbf{v}_{k} .
\end{aligned}
$$

In order to include a physical model for the DOT inverse problem, the model elements $\mathbf{A}, \mathbf{B}, \mathbf{C}$ and $\mathbf{D}$ are defined as

$$
\begin{aligned}
& \mathbf{A}=\mathbf{I}_{\left(n_{x}\right)} \\
& \mathbf{B}=\mathbf{I}_{\left(n_{u} n_{c} n_{g}\right)} \otimes\left[\begin{array}{ll}
\mathbf{0}_{\left(1, n_{r}-1\right)} & 0 \\
\mathbf{I}_{\left(n_{r}-1\right)} & \mathbf{0}_{\left(n_{r}-1,1\right)}
\end{array}\right] \\
& \mathbf{C}=\mathbf{I}_{\left(n_{u}\right)} \otimes \mathbf{1}_{\left(n_{c} n_{g}, 1\right)} \otimes\left[\begin{array}{ll}
\mathbf{1} & \left.\mathbf{0}_{\left(n_{r}-1,1\right)}\right]
\end{array}\right. \\
& \mathbf{S}=\mathbf{1}_{\left(1, n_{u}\right)} \otimes \mathbf{I}_{\left(n_{y}\right)} \\
& \mathbf{L}=\mathbf{I}_{\left(n_{u}\right)} \otimes \mathbf{L}_{0} \\
& \mathbf{G}=\mathbf{I}_{\left(n_{u} n_{w}\right)} \otimes \mathbf{G}_{0} \\
& \mathbf{E}=\mathbf{I}_{\left(n_{u}\right)} \otimes \mathbf{E}_{0} \otimes \mathbf{I}_{\left(n_{g}\right)} \\
& \mathbf{M}=\mathbf{I}_{\left(n_{u} n_{c} n_{g}\right)} \otimes \mathbf{1}_{\left(1, n_{r}\right)} \\
& \mathbf{H}=\mathbf{I}_{\left(n_{u} n_{c} n_{g}\right)} \otimes \mathbf{H}_{0} \\
& \mathbf{U}\left(\mathbf{z}_{k}\right)=\mathbf{1}_{\left(n_{u} n_{c} n_{g}, 1\right)} \mathbf{z}_{k}^{T} \odot \mathbf{M} \\
& \mathbf{D}\left(\mathbf{z}_{k}\right)=\operatorname{SLGEU}\left(\mathbf{z}_{\mathbf{k}}\right) \mathbf{H} \text {, }
\end{aligned}
$$

where $T$ is the transpose operator, $\otimes$ is the Kronecker tensor product, $\odot$ is termby-term array multiplication, $\mathbf{I}$ is the identity matrix, $\mathbf{1}$ is a matrix of ones, $\mathbf{0}$ is a matrix of zeros and matrix sizes are indicated with parenthetical subscripts. The submatrix $\mathbf{L}_{0}$ is a block diagonal matrix formed from meassurement by voxel average effective pathlengths for each wavelength as described by [10. The columns of $\mathbf{G}_{0}$ contain a set of spatial basis functions that can be used to reduce the number of states and/or impose spatial smoothing of the state estimates. Known optical extinction coefficients are contained in the wavelength by chromophore submatrix $\mathbf{E}_{0}$. The columns of $\mathbf{H}_{0}$ contain temporal basis functions to reduce the number of states and/or impose temporal smoothing.

The Kalman filter is a recursive solution to the state estimation problem for the discrete-time process described by equations 1, 2 and 3. The recursions require initialization of the state estimate $\hat{\mathbf{x}}_{0}$ and estimated state covariance $\hat{\mathbf{V}}_{0}$ and then proceed with the following prediction-correction algorithm

$$
\begin{aligned}
\hat{\mathbf{x}}_{k \mid k-1} & =\mathbf{A} \hat{\mathbf{x}}_{k-1 \mid k-1} \\
\mathbf{z}_{k \mid k-1} & =\mathbf{B} \mathbf{z}_{k-1 \mid k-1}+\mathbf{C u}_{k}
\end{aligned}
$$




$$
\begin{aligned}
& \hat{\mathbf{V}}_{k \mid k-1}=\mathbf{A}_{t} \hat{\mathbf{V}}_{k-1 \mid k-1} \mathbf{A}^{T}+\mathbf{Q} \\
& \mathbf{U}_{k}=\mathbf{1}_{\left(n_{u} n_{c} n_{g}, 1\right)} \mathbf{z}_{k \mid k-1}^{T} \odot \mathbf{M} \\
& \mathbf{D}_{k}=\mathbf{S L G E U} \mathbf{H}_{k} \mathbf{H} \\
& \mathbf{K}_{k}=\hat{\mathbf{V}}_{k \mid k-1} \mathbf{D}_{k}^{T}\left(\mathbf{D}_{k} \hat{\mathbf{V}}_{k \mid k-1} \mathbf{D}_{k}^{T}+\mathbf{R}\right)^{-1} \\
& \hat{\mathbf{x}}_{k \mid k}=\hat{\mathbf{x}}_{k \mid k-1}+\mathbf{K}_{t}\left(\mathbf{y}_{k}-\mathbf{D}_{k} \hat{\mathbf{x}}_{k \mid k-1}\right) \\
& \mathbf{z}_{k \mid k}=\mathbf{z}_{k \mid k-1} \\
& \hat{\mathbf{V}}_{k \mid k}=\hat{\mathbf{V}}_{k \mid k-1}-\mathbf{K}_{k} \mathbf{D}_{k} \hat{\mathbf{V}}_{k \mid k-1} .
\end{aligned}
$$

We designed an experiment to test the basic functionality of this proposed method. We combine real DOT data with a simulated functional response and then analyze the result with static deconvolution and the proposed Kalman filter method. This experiment allows us to compare the estimated responses with the "true" response. Only a single trial is examined so the results are mainly illustrative. Data was collected from a human subject who was instructed to sit quietly and breath freely. Measurements were taken with a continuous wave DOT instrument 11 then demodulated and down sampled to $1 \mathrm{~Hz}$. The measurements were high pass filtered in a forward then reverse direction with a 6 th order IIR Butterworth filter with a cutoff frequency of $0.05 \mathrm{~Hz}$ and zero phase distortion. This filtering removes slow physiology that is sufficiently outside the frequency range of interest for the hemodynamic response that it can be ignored. Shortterm variability including the respiratory sinus arrhythmia, Mayer waves and vasomotion remain after filtering. The photon fluence $\Phi(t, \lambda)$ was then converted to a change in optical density $\Delta \mathrm{OD}$

$$
\Delta \mathrm{OD}(t, \lambda)=\ln \left(\frac{\Phi(t, \lambda)}{\Phi_{0}(\lambda)}\right),
$$

where $\Phi_{0}$ is average detected photon fluence, $\Delta \mathrm{OD}(t, \lambda)$ are measurements $\mathbf{y}$ as a function of time $t$ and wavelength $\lambda$. The three model inputs contained in $\mathbf{u}$ were the Boolean stimulus time vector, the blood pressure (BP) and heart rate variability $(\mathrm{HRV})$ with normalized variances.

Data from only a single source fiber and three detector locations were included in the analysis. The three detectors were arranged about $2 \mathrm{~cm}$ apart in a row and the source was placed $3 \mathrm{~cm}$ away from the center detector and equidistant from the other two. Three voxels defined the tissue volume under the optical probes. Voxel 1 represented the scalp and was common to all the detectors. Voxels 2 and 3 represented two regions of the brain located under the scalp voxel. A simulated functional response was added into the baseline hemodynamics in voxel 2 . The stimulus paradigm was event related with a 12 to 18 second inter-stimulus interval over the 300 second trial. The model used to simulate the hemodynamics was one period of a raised cosine with a delay and amplitude set differently for the $\mathrm{HbO}$ and $\mathrm{HbR}$ functional responses. The simulated waveforms can be seen in the results figures.

The pathlength submatrix $\mathbf{L}_{0}$ was computed with a diffusion approximation to the transport equation for a semi-infinite medium [10]. An identity matrix 
was used for the spatial basis set $\mathbf{G}_{0}$ and a normalized Gaussian function was used for the temporal basis set $\mathbf{H}_{0}$. The standard deviation for the Gaussian function was fixed at 1.5 seconds and the means were separated by 1.5 seconds over the regression time. The same temporal basis set was used for the static deconvolution. The state update noise covariance $\mathbf{Q}$ only contained nonzero terms on the diagonal elements. Diagonal terms related to the functional response were set to $3 \times 10^{-6}$ and those related to BP and HRV were set to $10^{-5}$. This imbalance in state update noise caused the functional response model to evolve more slowly than the systemic physiological models. The measurement noise covariance matrix $\mathbf{R}$ was set to an identity scaled by $10^{-3}$. These variances act as regularization and were adjusted to stabilize the estimation scheme.

\section{Results}

The state estimates from the Kalman filter were propagated through the forward model to calculate the component of the measurements that relates to each input. An example result of this signal separation for the $890 \mathrm{~nm}$ measurement from detector 1 is shown in figure 1, The functional response to the stimulus only accounts for $2.8 \%$ of the variance in the measurement whereas BP and HRV account for $11.3 \%$ and $77.9 \%$ respectively. The sum of the modeled components accounts for over $99.9 \%$ of the variance in the measurement.

The results of the static deconvolution analysis to recover the functional hemodynamic response is shown in figure 2. The functional responses are clearly present in the estimates but are distorted by large physiological noise artifacts. Compared to the true hemodynamics, the $\mathrm{HbO}$ estimate resulted in $\mathrm{R}^{2}=0.78$, which is reasonably good considering that the physiological noise dominates the measurement. For the smaller HbR signal, $\mathrm{R}^{2}=0.57$ with the true hemodynam-

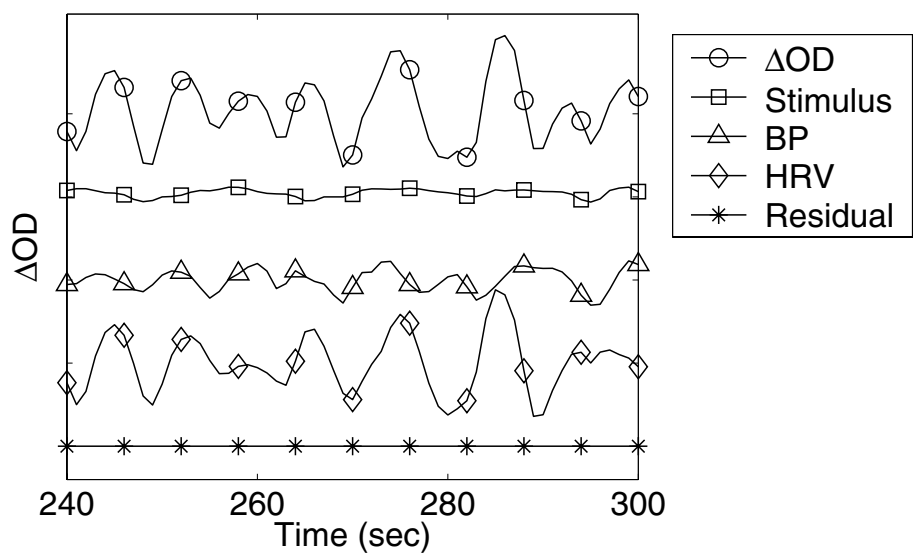

Fig. 1. Separating a $\triangle O D$ measurement into components related to each model input. The scale for each component of $\Delta \mathrm{OD}$ was shifted for visual comparison. 


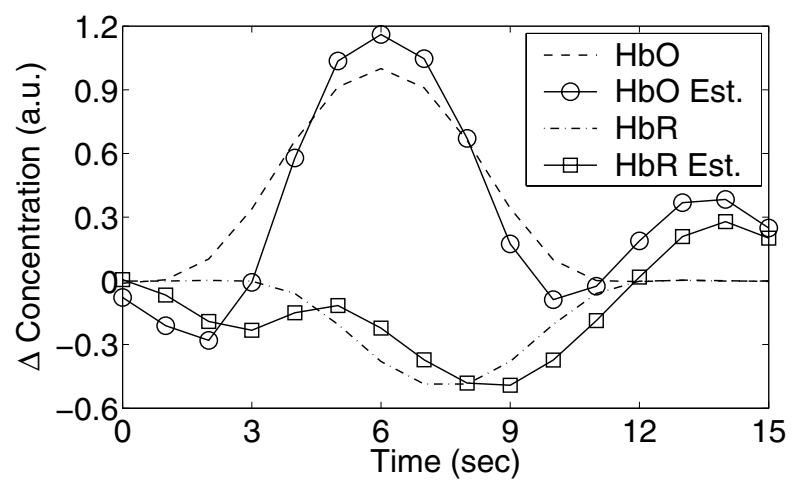

Fig. 2. Result for deconvolution of functional response from hemodynamics in voxel 2

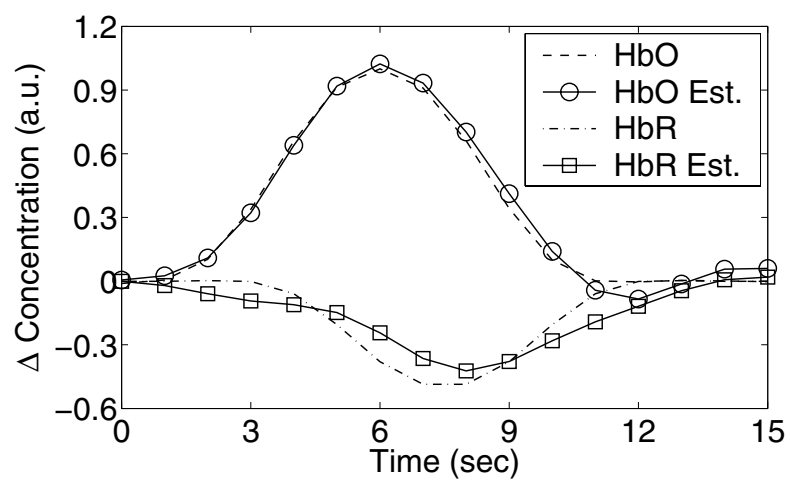

Fig. 3. Result for proposed Kalman filter estimate functional response in voxel 2

ics, indicating that the physiological noise artifacts are of comparable magnitude to the actual response.

The result for the Kalman filter was taken to be the last state estimate computed during a forward pass through the data. This result, shown in figure 3, appears to be a significant improvement over the deconvolution approach. The HbO estimate improved to $\mathrm{R}^{2}=0.99$ and the HbR estimate jumped to $\mathrm{R}^{2}=0.89$. There is some lag in the Kalman filter result which may have been caused by only using a forward pass through the data.

\section{Discussion}

We successfully implemented the Kalman filter for system identification in DOT. Based on the preliminary results described, the proposed analysis framework may help to improve estimates of functional hemodynamics in DOT neuroimaging. This result is potentially significant because improved hemodynamic estimates could make a broader range of brain activation paradigms possible with DOT. 
The ability to separate signals into physiological components may also reveal new information about the local regulatory physiology and may be useful in identifying certain vascular pathologies. Unlike the prior work with the Kalman filter for DOT, the present formulation has the flexibility to be applied to any experimental design and for problems of reasonably large spatial and temporal dimension. The proposed Kalman filter formulation may also be useful for other imaging modalities such as fMRI, MEG and EEG or when multiple modalities are combined with a single state-space model of the underlying physiology.

\section{References}

1. A.P. Gibson, J.H., Arridge, S.: Recent advances in diffuse optical imaging. Phys. Med. Biol. 50 (2005) R1-R43

2. Strangman, G., Culver, J., Thompson, J., Boas, D.: A quantitative comparison of simultaneous bold fMRI and NIRS recordings during functional brain activation. NeuroImage 17 (2002) 719-731

3. Kolehmainen, V., Prince, S., Arridge, S., Kaipio, J.: State-estimation approach to the nonstationary optical tomography problem. J. Opt. Soc. Am. A 20 (2003) $876-889$

4. Prince, S., Kolehmainen, V., Kaipio, J., Franceschini, M., Boas, D., Arridge, S.: Time-series estimation of biological factors in optical diffusion tomography. Phys. Med. Biol. 48 (2003) 1491-1504

5. Zhang, Y., Brooks, D., Franceschini, M., Boas, D.: Eigenvector-based spatial filtering for reduction of physiological interference in diffuse optical imaging. Journal of Biomedical Optics 10 (2005) 011014-1-11

6. Cohen, M.A., Taylor, J.A.: Short-term cardiovascular oscillations in man: measuring and modelling the physiologies. Journal of Physiology 542.3 (2002) 669-683

7. Frackowiak, R., Friston, K., Frith, C., Dolan, R., Price, C., Zeki, S., Ashburner, J., Penny, W., eds.: Human Brain Function. 2nd edn. Academic Press (2003)

8. Roche, A., Pinel, P., Dehaene, S., Poline, J.: Solving incrementally the fitting and detection problems in fMRI time series. Medical Image Computing and ComputerAssisted Intervention (MICCAI'04), Part 2, Lecture Notes in Computer Science $\mathbf{3 2 1 7}$ (2004) 719-726

9. Kalman, R.: A new approach to linear filtering and prediction problems. Trans. of the ASME-Journal of Basic Engineering 82 (1960) 35-45

10. Arridge, S., Cope, M., Delpy, D.: The theoretical basis for the determination of optical pathlengths in tissue: temporal and frequency analysis. Phys. Med. Biol. 37 (1992) 1531-1560

11. Franceschini, M., Fantini, S., Thompson, J., Culver, J., Boas, D.: Hemodynamic evoked response of the sensorimotor cortex measured non-invasively with nearinfrared optical imaging. Psychophysiology 40 (2003) 548-560 